\title{
A review of mud pulse telemetry signal impairments modeling and suppression methods
}

\author{
Saleh M. Mwachaka ${ }^{1,2} \cdot$ Aiping $\mathrm{Wu}^{1} \odot$ - Qingqing $\mathrm{Fu}^{1}$
}

Received: 7 November 2017 / Accepted: 10 May 2018 / Published online: 2 June 2018

(c) The Author(s) 2018

\begin{abstract}
Exploration and drilling operations are the most costly and risky activities that require logging while drilling effective tool operation and high speed real-time data transmission. Among other logging technologies, mud pulse telemetry has been widely adopted into real-time drilling and logging data measurements for deep formation evaluation decision-making. However, the increased downhole information quantity and quality requirements have elevated demands for greater bandwidth and higher data rate transmission. Maximum possible mud pulse telemetry data rates are obstructed by unpredictable signal impairments. Researchers have implemented numerous downhole signal modulation techniques, data compression algorithms and surface receiver noise cancellation techniques to lower bit errors and increase signal-to-noise ratio. This study reviews mud pulse telemetry system signal transmission challenges and current countermeasure trends. Rigorous technical literature reviews from exploration and drilling oilfield service industry have been at the receiving end debated. Mud channel signal characteristics, signal attenuation factors, signal detection and decoding techniques are critical to successful mud pulse operations. To minimize exploration, drilling and economic risks, this study explores mud pulse data transmission challenges, strategies and possible technical solutions to the next generation engineers and scholars in the field.
\end{abstract}

Keywords LWD $\cdot$ Mud pulse telemetry $\cdot$ Signal impairments $\cdot$ Noise suppression methods

\section{Introduction}

Drilling operations account for the largest percentage of expenses incurred in the exploration and development of oil and gas reservoirs (de Almeida Jr et al. 2015). The economic impact of the exploratory drilling activity accounts for $4-16 \%$ of the total field cost, making it necessary to have accurate drive and control operation from unpredicted events. Accurate real-time drilling information collected by more sensors determine wellbore directional drilling variables and geological formation data critical for formation evaluation and drilling efficiency (Liu et al. 2007). Logging information provide drill bit inclination and azimuth direction, natural gamma ray radiation levels and rock formation

Aiping Wu

wuaping@yangtzeu.edu.cn

1 School of Electronic Information, Yangtze University, Jingzhou 434023, Hubei, China

2 Faculty of Computing, Information Systems and Mathematics, Institute of Finance Management, 3918 Dar es Salaam, Tanzania electrical resistivity highly need for decision-making. Exploration and drilling activities have long acknowledged the need for high-speed real-time data rate technologies driven by several factors which include high density logging data, safety real-time drilling information and managing economic risk by enabling more accurate information for formation evaluation ( $\mathrm{Su}$ et al. 2011). Faster downhole to surface data transmission systems requires high-speed realtime data transmission that will not only improve the drilling efficiency, but also will greatly reduce the risk of any other engineering accident (Akimov et al. 2006; Hartmann et al. 2012).

The increased complexity and cost of oil and gas exploration and drilling industry depends on logging-while-drilling (LWD) technology. The term LWD encompasses equipment and techniques for real-time data transmission from within the well to the earth's surface detectors (Shi et al. 2004). LWD systems are responsible for making various downhole measurements and transmits the collected information to the surface for display, interpretation and immediate use (Shao et al. 2017). In today's drilling, mud pulse telemetry (MPT) system is the common low-cost technology that provides 
reliable two-way real-time data transmission between the wellbore downhole system and the surface receiver (Hutin et al. 2001; Liu et al. 2007). For more than a decade, MPT has gained advantages in downhole data transmission technology that includes the downhole mud pulse generator and ground signal detection and extraction system ( $\mathrm{Su}$ et al. 2011). Proper well conditioning, navigation, and feedback control for real-time target relocation requires more sophisticated technical solutions to improve drilling efficiency, safety, and best scoping of the best hydrocarbon production zones. The availability of different telemetry systems design improvements has allowed the industry to overcome diverse challenges facing transmission of information from downhole to the surface using varying techniques and methods.

However to date, the challenges facing LWD mud pulse signal transmission characteristics still require extensive research. Academicians and researchers are not discussing similar techniques on the effects of various sources of signal impairments and methods reducing and eliminating them. Furthermore, results from empirical studies are still diverse and contradicting. In this study, compilation of technical articles, papers, journals and patents, has been used to gain theoretical background, technical understanding and industry practical applicability of MPT system operations and related challenges.

The study reviews various signal impairments that affect the quantity and quality of mud pulse telemetry signal required to maximize real-time data transmission throughput between downhole system and surface receiver. The paper explores mud pulse signal transmission and processing technical solutions to overcome limitations from downhole signal generation, signal transmission and impairments and signal detection and extraction at the surface receiver. The paper is organized into eight (8) sections: The next section explores the LWD technologies while the subsequent section reviews the mud pulse telemetry system signal generation, transmission, detection and extraction; then the following section discusses the various sources of signal impairments affecting the mud pulse signal transmission and extraction; subsequently, the current techniques and methods applied to suppress unwanted mud pulse signal impairment effects are reviewed; before summarizing the paper, comprehensive MPT technological performance advances are reviewed; thereafter, the paper is summarized and discussed; finally, the paper concludes.

\section{LWD telemetry systems}

This section reviews a number of common logging while drilling (LWD) technologies in the oil and gas drilling operations. The review aims at identifying the benefits of faster and better quality telemetry systems as the basis of effective geo-steering decision making and drilling risk control (Wassermann et al. 2009). Downhole telemetry technologies that transfer drilling data to the surface are categories into wired-pipe telemetry (WPT) and wireless telemetry systems that include electromagnetic (EM), acoustic and mud pulse (MPT) telemetry systems (Hutin et al. 2001). Increased downhole collected information quantity have led the deployment of telemetry technology to be limited by transmission bandwidth of critical data (Nygard et al. 2008). The increased bandwidth demand guarantee complete drilling and formation evaluation in real time (Wolfe et al. 2009). The real-time data are important for well construction analysis and decision making to optimize drilling and well completion strategies while drilling.

Early researches were passionate about electromagnetic (EM) telemetry technologies to transmit downhole drilled information with data rates reaching above 1 bps being realized over significant depths, and claimed the possibility of reaching $100 \mathrm{bps}$ with repeater system (Harrison et al. 1990). EM telemetry was found to be a reliable system as it lacks moving parts, but had limitations on the types of wells where it can be used. The electromagnetic waves conducting medium decays the electromagnetic signal and limit the signal propagation to impractical distances (Neff et al. 2007). However, mud pulse telemetry advanced in parallel to EM and today's mud pulse systems are capable of greater than 20 bps from depths greater than $6096 \mathrm{~m}$ with repeater less systems (Wasserman et al. 2008). The EM telemetry high electrical conductivity was found imperfect in water depths offshore operations, relegated the technology to oil and gas market onshore, shallow depths that need low transmission rates (Schnitger et al. 2009). In the end, electromagnetic telemetry technology growth was declined due to high attenuation suffered by the electromagnetic signals in water environments and limited transmission distance.

Acoustic telemetry technology transmission performance is not mature enough; mostly applied in exploratory and testing wells operation limited to shallow depth wells of less than $2500 \mathrm{~m}(<2500 \mathrm{~m})$ (de Almeida Jr et al. 2015). Mud pulse telemetry technology uses the modulated mud pressure signal pulses that traverse along the drilling pipe to deliver data at the surface over a long transmission distance exceeding $10,700 \mathrm{~m}$. The acoustic telemetry unlimited depth capability can be realized when acoustic repeater is deployed (Neff et al. 2007), adding extra maintenance cost. This system transmission waves have many harmonics that propagate through the pipe string which experience a decent frequency-dependent decay while propagating causing noticeable signal dispersion due to multipath reflections in the pipe string (Farraj et al. 2013).

The introduction of wired-pipe technology (WPT) was first tested in 2003 and commercially launched in 2006 (Ali et al. 2008; Reeves et al. 2006), offered the wider real-time 
bandwidth (Manning et al. 2007), allowing reliable, bi-directional data transmission at speeds up to $57,600 \mathrm{bps}$ (Hernandez et al. 2008). The wired CTT technology hindered the conventional mud pulse and electromagnetic telemetry method bandwidth restriction and time lag, resulting into high speed data transmission systems. WPT systems massively expanded the quantity and quality of information available while drilling giving much greater volume, resolution and quality of formation evaluation and drilling dynamics data reducing the non-productive time (NPT) (Nygard et al. 2008).

The most advanced coiled tubing telemetry (CTT) systems that have been extensively used employ either the $1 / 8^{-}$ inch insulated electrical conductor (Livescu et al. 2015a, b; Taggart et al. 2011), or the optical fiber (Mokhtar et al. 2014; Ramondenc et al. 2013). The functionality and capabilities of both wired CTT types bottomhole assemblies (BHAs) systems are designed to meet similar requirements only differs on how the electrical power is provided to the downhole sensors (Livescu et al. 2015a). The insulated electrical wired CTT system transfer electrical power through its electrical conductor while optical fiber CTT system requires BHA batteries to power its downhole sensors. The common insulated electrical conductor BHA sizes include $2 \frac{1}{8^{-}}, 2^{7} / 8^{-}$, and $3 \frac{1}{2}$-inches designed with several single-point sensors for real-time measurements of downhole pressure, temperature, and location (Blanco et al. 2017). CTT systems field test with depth, pressure, and temperature data in real-time increased the job efficiency and well intervention control operations without mobilizing the crew, wireline and tractor equipment, saving an estimated of six (6) days minimum per well wireline logistics and work.

Due to their advanced design and operational complexity, coiled tubing telemetry systems present several design challenges. The large volume of real-time data acquired by CTT downhole system still requires image compression before data transmission. The image compression ratios achieved with WPT systems are significantly lower compared to ratios achieved by MPT systems yielding to unmatched resolution of close-to-memory image definition (Wolfe et al. 2009). CTT operation records low signals which contain high noise signals that need proper noise cancellation techniques for accurate readings; small space installed printed circuit boards (PCBs) due to the annular flow path comprise many interconnections that increase the design complexity and power consumption; the annular ring installation contains foil strain gauges that are permanently glued with heat-cured epoxy resin on the inside wall of its pressure housing, thus wiring mounting requires a specialized tool and a highly skilled technician and time consuming (Blanco et al. 2016). The initial CTT assembly laboratory test is performed at atmospheric and downhole pulling and pushing pressure force conditions that require complex differential pressure mathematical modeling for pressure compensation at downhole condition (Garner et al. 2016). Currently, the complex quantitative interpretation model for acid treatments required for qualitative interpretation and surface display of wired CTT downhole data in real time is yet undisclosed (Blanco et al. 2017).

The recent MPT technology advancements marked a stepchange improvement in providing real-time data rates from greater depths, whereby currently available MPT system can achieve up to 20 bps (Wasserman et al. 2008). The combined high data rate techniques with state-of-the-art image compression technologies have led mud pulse telemetry appropriate for fine-scaled geological feature determination (Fulda et al. 2006). Table 1, summarizes the performance of the different LWD telemetry technologies. Mud pulse demonstrates reliable data transmission performance records, feasible to wide range operating environments and low cost operations.

\section{Mud pulse telemetry (MPT) system}

Modern petroleum exploration, drilling and production operations demand great information quantity relating to downhole parameters and conditions that contain earth formation characteristics traversed along the drilling borehole (Shearer 2010). LWD technology has ordinarily employed mud pulse telemetry (MPT) systems to measure those parameters and increase field operations' profit and economic value. The MPT system transmits signals using the communication channel which consists of drilling fluid column, normally the mud, which runs in the drill pipe between downhole
Table 1 Comparative performance among LWD telemetry technologies. (Source: Compiled from literatures)

\begin{tabular}{lllll}
\hline Features & \multicolumn{2}{l}{ LWD telemetry technology } & \\
\cline { 2 - 5 } & Electromagnetic & Acoustics & Mud pulses & Wired drill pipe \\
\hline Max. transmission data rate (bps) & 10 & 20 & 20 & 57,600 \\
Maximum depth (meters) & 5500 & 3700 & 12,200 & Unlimited \\
Data quantity & Medium & Low & High & Very high \\
Signal attenuation & High & High & Medium & N/A \\
Signal interference & High & Medium & Medium & Low \\
Installation and other cost & Medium & Medium & Low & High \\
\hline
\end{tabular}


transmitter and the surface receiver (Klotz et al. 2007, 2008a). The drilling fluid is used to lubricate and cool downhole drillstring components, carry cuttings from the base of the borehole to the surface, and balance the hydrostatic pressure in the rock formations. MPT system uses coded drilling fluid pressure signal pulses generated at downhole that propagate through the mud to uphole surface system where are detected and decoded for interpretation (Hutin 2012). The downhole drilling fluid passes through a moving valve that restricts mud flow and in turn generates pressure waves which travel to surface at varying speeds depending on the drilling fluid properties. Controlled pressure pulse variations at downhole are used to modulate amplitude, phase and frequency of mud pulse signals generated by mud pumps (Hahn et al. 2008). The surface received signal is responsive to the pulse variations related to the downhole measured data. To send a stream of data, three known mud pulse pressure pulses, positive-pulse, negative-pulse (Annular-venting telemetry) and continuous-wave (mud siren) waves are generated, Fig. 1 (Hutin et al. 2001). The pressure pulses generated by poppet pulsers form positive-pulse and negative-pulse (annular-venting telemetry) systems that are discrete pressure waves; siren pulsers generate continuous wave pulses that are periodic; and rotationally oscillating shear-valve pulsers that may generate discrete pulses and/or continuous wave signals (Shi et al. 2004). These generated mud pulsar series are recognizable by the surface signalprocessing module (Shi et al. 2004). Details on generation of these pressure pulses can be read from (Hahn et al. 2008; Hutin 2012; Reckmann 2014; Shi et al. 2004) scientific research works.

Transmitted pulse signals containing critical well drilling information are encoded by various techniques in advance before they propagate to surface for detection and decoding (Li and Reckmann 2009). The surface pressure transducers detect signals weakened by signal strength degradation (attenuation), erosion, pressure drop across the borehole and other signal impairment effects (Hahn et al. 2008; Hutin 2012). The received information contains logging data and parameters of pressure, temperature, drill bit direction and deviation. Critical logging include electrical conductivity of the various formation layers, acoustic and nuclear properties, porosity, induction, and pressure gradients (Klotz et al. 2007). To improve drilling operations and economic production of the reservoirs, methods to recover the downhole transmitted signals are mandatory.

\section{MPT system signal impairments}

Mud channel signals experience pressure fluctuations and uncertainty caused by various MPT system components that potentially change signal properties during the course of data transmission. The main noise sources that induce pressure fluctuations are mud pumps, pulsation dampeners, surface piping, pressure transducer locations, drill string components, mud properties and the well depth (Klotz et al. 2008a, c). The mud channel that the pulse signals pass as they propagate to the surface is extremely harsh, demanding complex surface signal detection and extraction. Common mud pulse signal property distortions which degrade signal transmission and data rates are downhole signal strength, signal attenuation, surface induced noise (electrical noise) and surface piping induced signal reflections (Klotz et al. 2008c). Unpredictable and complex adjustable signal impairments are caused by drilling noise, drill string motion noise within the borehole, attenuation and circulation pumps noise (Shearer 2010). Depending on the severity of the mud channel conditions and signal distortions, to achieve maximum possible data rates, telemetry systems must be robust and flexible, both downhole and at the surface receiver (Klotz et al. 2008a, b, c). Despite the signal processing complexity and their varying properties, mud pulse systems must adapt the harsh working environment and recover the useful original signals. This section discusses main mud pulse telemetry system signal impairments that affect MPT data transmissions.

\section{MPT mud pump noises}

During mud pulse signal transmission, mud pumps generate down-going mud flow which creates pressure signals
Fig. 1 Generated mud pulses: positive (a), negative (b) and continuous (c)

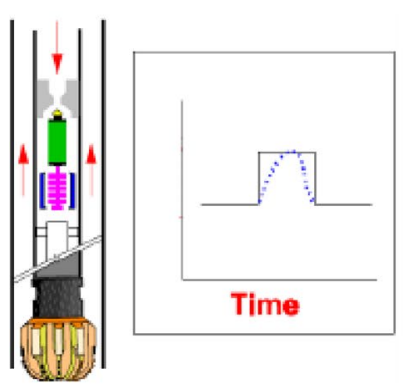

(a)
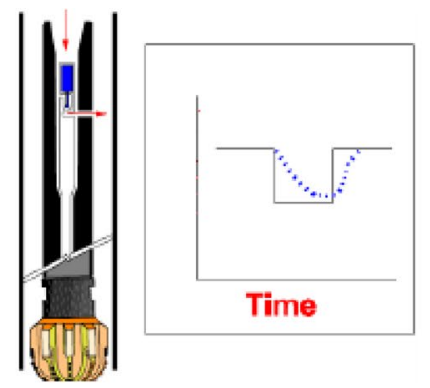

(b)

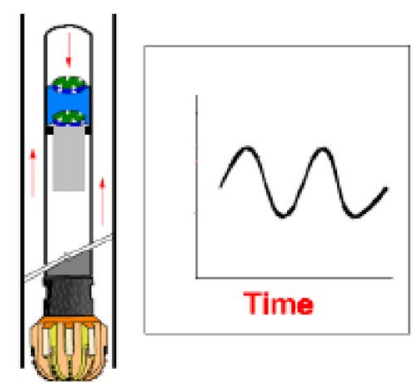

(c) 
by simultaneously opening and closing intake and exhaust valves and moving a piston back and forward within a cylinder. Reciprocating motion of mud pump pistons generates pump signals propagating downstream in opposite direction from the downhole pulse information carrying signal wave (Hahn et al. 2008). Forcing mud through the exhaust valve create mud pressure variations which pass through the surface transducer down the drill string to the drill bit and results into cumbersome mud pump noise signals (Reckmann 2014). Despite pressure random noise and signal interferences, generated mud pump pressure fluctuations create notable signal amplitude and frequency variations within the frequency ranges $1-20 \mathrm{~Hz}$, commonly used for LWD data telemetry systems (Brackel 2016; Li and Reckmann 2009; Shen et al. 2013a, b). The MPT pulse signal is lost in the pump noise which affects surface pressure transducers as are located close to mud pumps than to the mud pressure pulsar generators (Brackel 2016; Brooks 2015). Mud pump noises have frequency magnitude greater than the original pulse pressure signal waves, causing difficult correct signal extractions.

The frequencies at which pump noise tones occur are harmonic in nature and appears as set of frequency integers that are multiples of pump stroke rates fundamental frequency, e.g., 3F, 6F, 9F, etc, that can be estimated by Eq. 1 . The amplitudes of the dominant harmonics are higher than other harmonics, if selected will affect the signal energy leading to demodulation and data-decoding complex (Hutin et al. 2001). To minimize the effect of this noise, it is possible to predict on which frequency the harmonics will appear using the following equation:

$f_{n}=(n \times s) / 60$,

where $f_{n}$ is the frequency of the $n$th harmonic. $s$ is the pump stroke rate, stroke/minute, and $n$ is the Harmonic number, $n \in\{1,2,3, \ldots\}$.

To diminish the effect of the individual contributions of each piston in a mud pump, MPT system use pulsation dampeners to smooth the pressure pulses. However, certain pressure signal objects remain and may distort the pressure pulse signals (Brackel 2016). The dominant pump harmonics which have high-energy signatures with potential to generate significant distortion to the telemetry can be estimated using the following equation:

$n=m \times N_{c} \times a$,

where $n$ is the harmonic number, $m$ is the positive integer number, $N \mathrm{c}$ is the number of cylinders in the pump s, and $a$ is the pump action $(1=$ single, $2=$ double $)$.

Methods to remove mud pump noises and reflections from the measured signal use two spaced transducers at surface receivers. The measured signal at first transducer is delayed by the approximated time that takes the pressure pulses to travel between transducers and later subtracted from the signal measured at the second transducer (Reckmann 2008; Wassermann et al. 2008). Signal processing of the received signal from first and second transducers is added with active pump strobe signal and used to obtain pump signatures at first and second spaced transducer measurements. The signal transfer function is computed from channel pump signatures and used to estimate the downhole transmitted telemetry signal. Initially, attempts to solve the problem used the received signals to determine signal transfer function of the communication channel between two transducers (Reckmann 2014; Wassermann et al. 2008). The estimated received signals transfer function can be unreliable, inaccurate and misrepresentation of the signal attenuation and/or distortion as it contains interfered signal components of downhole up-going signal and mud pump down-going signal directions (Wassermann et al. 2008). Least mean-squares filtering algorithm was used to reduce pump noise signals based on frequency characteristics analysis of the noise generated by one or more pumps ( $\mathrm{Li}$ and Reckmann 2009; Shao et al. 2017). The frequency domain analysis of the pump frequencies is fine-tuned in the time domain and a synthetic timing signal is used for the filtering.

\section{MPT signal attenuation and dispersion}

As MPT system signals propagate through the drilling mud along the borehole, signals are attenuated and dispersed. This is caused by underbalanced drilling mud which results into viscous dissipation and frictional energy loss at the borehole walls (Harrell et al. 2000). To minimize reservoir damage, the drilling mud pressure is kept high that exceeds the hydrostatic pressure and nitrogen gas is used to reduce mud density. The effect increases the drilling mud compressibility hence reducing mud bulk modulus and mud pulse propagation velocity in the drilling fluid (Lin et al. 2013). Changes in drilling mud compressibility may result into serious earth formation damage which strongly affects the shape of the signal pulses and complicates the decoding process. Factors influencing mud pulse signal attenuation include the borehole depth, drilling mud type, number of joints in the drill string, drillstring inner diameter and signal operating frequency (Brooks 2015). Attenuation impacts may not be constant across the range of frequency components, lower frequency components are subject to less attenuation than higher frequencies (Klotz et al. 2008c). Devising good control of the various noise sources in $1-20 \mathrm{~Hz}$ frequency band, it is advantageous to transmit MPT data at the lowest possible frequency to minimize the signal attenuation impacts. In high data rate exploration and drilling operation applications, the effect of signal attenuation is not the major burden but rather the various noise sources and their distribution across the available channel transmission band. 


\section{Signal echoes and reflections}

High data rate pulsars in MPT telemetry not only create signals that propagate uphole, but also reverse direction, down-going signals that reflect at the drillbit which combine to create constructively or destructively interfering signals. Strong signal reflections are detected at the surface signal detectors caused by mud pumps, pulsation dampeners, drill string joints and diameter changes, dispersion and filtering of certain frequencies within mud channel (Brackel 2016; Brooks 2015). The ultimate signal that travels up the drillpipe depends on mud velocity, the position of the pulsar in the drill collar, operating frequency and the bottomhole assembly details forming the host waveguide. The superposition of the main wave from the transmitter and the multiple reflected waves generate either destructive interference or constructive interference pulse signals (Shi et al. 2004). Destructive interference signal can severely limit data rate and transmission distance while the constructive interference signal, if properly applied, can enhance LWD signal strength without the power penalties incurred by mechanical methods. As a matter of fact, signal reflections occur at any impedance mismatch positions in drillstring, reducing its impacts (Cheng et al. 2014a, b) proposed novel multipleecho suppression model using two receivers based on the transient pulse responses in uplink and downlink channels.

\section{Drill string rock formation and gas}

The rock formation solid particles, free gas volume fraction and mud channel compressibility significantly affect the mud pulse velocity and signal attenuation ( $\mathrm{Li}$ et al. 2015; Liu et al. 2007). Solid particles caused by the rock formation that are destroyed during the drilling process, form formation fragments of different sizes and the gas may enter the borehole when a high-pressure zone is being penetrated (Cowan 2005; Lu et al. 2013). As mud solids and the free gas content increases, mud density and mud compressibility changes decrease the mud pulse velocity which add complexity to signal transmission speed (Liu et al. 2007; Shi and Liu 2002). The initial mud pulse propagation velocity calculation models considered drilling mud to be a single phase; however, drilling mud is a multiphase, containing clay, formation cuttings of various sizes, barite, and free gas mixed with water or oil, all affecting the mud bulk modulus (Shi and Liu 2002). Drilling mud borehole boundary constraint study using mud pulse velocity continuity derived equation shows that water-based mud velocity is generally higher than oil-based mud. If gas influx is not offset in time, the unstable effect can escalate into well blowout creating severe financial losses, environmental contamination, and even potential human lives loss (Lin et al. 2013).

\section{Downhole random noise (electrical noise)}

Downhole sources of random noise are the pressure fluctuations caused by bit vibration, drilling motor stalling and the drill bit interaction with formation being drilled. Mechanical rig vibration and electrical noise coupling into the electrical wiring that carries the electrical signals from the drilling sensors to the signal receiver may degrade telemetry signal detection and extraction (Shi et al. 2004). These noises present the band-limited white Gaussian noise due to the lower noise frequency spectrum (Hutin et al. 2001). Although the random noise frequencies are small but they can affect certain signal frequency bands, causing relatively larger random pressure amplitude fluctuations and may lower downhole signal-to-noise ratio (SNR).

Signal impairments degrade the quality of the surface received signal and complicate telemetry signal detection and extraction. Consequently, methods and techniques to recover the transmitted downhole signal at the surface are becoming key problems for LWD mud pulse systems.

\section{Signal impairment suppression methods}

Researchers have shown that mud pulse telemetry technologies have gained exploration and drilling application advantages by providing cost-effective real-time data transmission in closed-loop drilling operations. Given the inherited mud pulse operation difficulties, there have been numerous communication channel efforts to improve data rate speed and transmission distance in LWD operations. As discussed in "MPT systems signal impairments", mud pulse signal pulse transmissions are subjected to mud pump noise signals, signal attenuation and dispersion, downhole random (electrical) noises, signal echoes and reflections, drillstring rock formation and gas effects, that demand complex surface signal detection and extraction processes. A number of enhanced signal processing techniques and methods to signal coding and decoding, data compression, noise cancellation and channel equalization have led to improved MPT performance in tests and field applications. This section discusses signal-processing techniques to minimize or eliminate signal impairments on mud pulse telemetry system.

\section{Matched filter}

At early stages of mud pulse telemetry applications, matched filter demonstrated the ability to detect mud pulse signals in the presence of simulated or real noise. Matched filter method eliminated the mud noise effects by calculating the self-correlation coefficients of received signal mixed with noise (Marsh et al. 1988). Sharp cutoff low-pass filter was proposed to remove mud pump high frequencies and 
improve surface signal detection. However, matched filter method was appropriate only for limited single frequency signal modulated by frequency-shift keying (FSK) with low transmission efficiency and could not work for frequency band signals modulated by phase shift keying (PSK) (Shen et al. 2013a).

\section{Wavelet transform}

Wavelet transform method was developed and widely adopted and used in signal processing to overcome limitation of Fourier transform in time domain (Bultheel 2003). Although Fourier and its revised fast Fourier transforms are powerful mathematical tool, they are not very good at detecting rapid changes in signals such as seismic data and well test data in petroleum industry containing many structure of different scales (Multi-scale structures) (Guan et al. 2004). Fourier coefficients do not provide direct information about the signal local behavior (localization); but the average strength of that frequency in the full signal as the sine or cosine function keeps undulating to infinity. Wavelet transform analyzes the signal frequency components and time segment, and fine tune sampling of localized characters of time or frequency domain. Principles of wavelet transform and de-noising technique show that signal can be divided into space and scale (time and frequency) without losing any useful information of the original signal, hence ensuring the extraction of useful information from the noised signal ( $\mathrm{Li}$ et al. 2007). Different wavelet base parameters constructed, such as haar, db, coif, sym, bior, rbio and dmey, are suitable for different signal processing requirements. The small the scale parameter is, the higher the resolving power in frequency, suitable for processing high frequency signals; conversely, the larger the scale is the higher resolving power suitable for low frequency signal.

In processing noise-contaminated mud pulse signals, longer vanishing moments are used, but takes longer time for wavelet transform. The main wavelet transform method challenges include effective selection of wavelet base, scale parameters and vanishing moment; the key determinants of signal correlation coefficients used to evaluate similarities between original and processed signals. Chen et al. (2010) researched on wavelet transform and de-noising technique to obtain mud pulse signals waveform shaping and signal extraction based on the pulse-code information processing to restore pulse signal and improve SNR. Simulated discrete wavelet transform showed effective de-noise technique, downhole signal was recovered and decoded with low error rate. Namuq et al. (2013) studied mud pulse signal detection and characterization technique of non-stationary continuous pressure pulses generated by the mud siren based on the continuous Morlet wavelet transformation. In this method, generated non-stationary sinusoidal pressure pulses with varying amplitudes and frequencies used ASK and FSK modulation schemes. Simulated wavelet technique showed appropriate results for dynamic signal characteristics analysis.

While Fourier coefficients provide average signal information in frequency domain and unable to reveal the nonstationary signal characteristics, wavelet transform can effectively eliminate MPT random noise when signal carrier frequency characteristics (periods, frequencies, and start and end time) are carefully analyzed.

\section{Adaptive noise-filtering techniques}

As discussed in "MPT mud pump noises", the often overlap of the mud pulses frequency spectra with the mud pump noise frequency components adds complexity to mud pulse signal detection and extraction. Real-time monitoring requirement and the non-stationary frequency characteristics made the utilization of traditional noise filtering techniques very difficult (Brandon et al. 1999). The MPT operations practical problem contains spurious frequency peaks or outliers that the standard filter design cannot effectively eliminate without the possibility of destroying some data. Therefore, to separate noise components from signal components, new filtering algorithms are compulsory.

Early development Brandon et al. (1999) proposed adaptive compensation method that use non-linear digital gain and signal averaging in the reference channel to eliminate the noise components in the primary channel. In this method, synthesized mud pulse signal and mud pump noise were generated and tested to examine the real-time digital adaptive compensation applicability. However, the method was not successfully applied due to complex noise signals where the power and the phases of the pump noises are not the same.

Jianhui et al. (2007) researched the use of two-step filtering algorithms to eliminate mud pulse signal direct current (DC) noise components and attenuate the high frequency noises. In the study, the low-pass finite impulse response (FIR) filter design was used as the DC estimator to get a zero mean signal from the received pressure waveforms while the band-pass filter was used to eliminate out-of-band mud pump frequency components. This method used center-ofgravity technique to obtain mud pulse positions of downhole signal modulated by pulse positioning modulation (PPM) scheme. Later Zhao et al. (2009) used the average filtering algorithm to decay DC noise components and a windowed limited impulse response (FIR) algorithm deployed to filter high frequency noise. Yuan and Gong (2011) studied the use of directional difference filter and band-pass filter methods to remove noise on the continuous mud pulse differential binary phase shift keying (DBPSK) modulated downhole signal. In this technique, the directional difference filter was

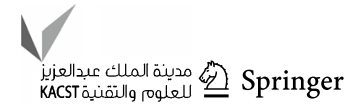


used to eliminate mud pump and reflection noise signals in time domain while band-pass filter isolated out-of-band noise frequencies in frequency domain.

Other researchers implemented adaptive FIR digital filter using least mean square (LMS) evaluation criterion to realize the filter performances to eliminate random noise frequencies and reconstruct mud pulse signals. This technique was adopted to reduce mud pump noise and improve surface received telemetry signal detection and reliability. However, the quality of reconstructed signal depends on the signal distortion factor, which relates to the filter step-size factor. Reasonably, chosen filter step-size factor reduces the signal distortion quality. Li and Reckmann (2009) research used the reference signal fundamental frequencies and simulated mud pump harmonic frequencies passed through the LMS filter design to adaptively track pump noises. This method reduced the pump noise signals by subtracting the pump noise approximation from the received telemetry signal. Shen et al. (2013a) studied the impacts of filter step-size on signal-to-noise ratio (SNR) distortions. The study used the LMS control algorithm to adjust the adaptive filter weight coefficients on mud pulse signal modulated by differential phase shift keying (DPSK). In this technique, the same filter step-size factor numerical calculations showed that the distortion factor of reconstructed mud pressure QPSK signal is smaller than that of the mud pressure DPSK signal.

Study on electromagnetic LWD receiver's ability to extract weak signals from large amounts of well site noise using the adaptive LMS iterative algorithm was done by (Liu 2016). Though the method is complex and not straightforward to implement, successive LMS adaptive iterations produced the LMS filter output that converges to an acceptable harmonic pump noise approximation. Researchers' experimental and simulated results show that the modified LMS algorithm has faster convergence speed, smaller steady state and lower excess mean square error. Studies have shown that adaptive FIR LMS noise cancellation algorithm is a feasible effective technique to recover useful surface-decoded signal with reasonable information quantity and low error rate.

\section{Other methods}

Different techniques which utilize two pressure sensors have been proposed to reduce or eliminate mud pump noises and recover downhole telemetry signals. During mud pressure signal generation, activated pulsar provides an uplink signal at the downhole location and the at least two sensor measurements are used to estimate the mud channel transfer function (Reckmann 2008). The telemetry signal and the first signal (pressure signal or flow rate signal) are used to activate the pulsar and provide an uplink signal at the downhole location; second signal received at the surface detectors is processed to estimate the telemetry signal; a third signal responsive to the uplink signal at a location near the downhole location is measured (Brackel 2016; Brooks 2015; Reckmann 2008, 2014). The filtering process uses the time delay between first and third signals to estimate the two signal cross-correlation (Reckmann 2014). In this method, the derived filter estimates the transfer function of the communication channel between the pressure sensor locations proximate to the mud pump noise source signals. The digital pump stroke is used to generate pump noise signal source at a sampling rate that is less than the selected receiver signal (Brackel 2016). This technique is complex as it is difficult to estimate accurately the phase difference required to give quantifiable time delay between the pump sensor and pressure sensor signals.

As mud pulse frequencies coincide with pump noise frequency in the MPT $1-20 \mathrm{~Hz}$ frequency operations, applications of narrow-band filter cannot effectively eliminate pump noises. Shao et al. (2017) proposed continuous mud pulse signal extraction method using dual sensor differential signal algorithm; the signal was modulated by the binary frequency-shift keying (BFSK). Based on opposite propagation direction between the downhole mud pulses and pump noises, analysis of signal convolution and Fourier transform theory signal processing methods can cancel pump noise signals using Eqs. 3 and 4. The extracted mud pulse telemetry signal in frequency domain is given by Eqs. 3 and 4 and its inverse Fourier transformation by Eq. 4 . The method is feasible to solve the problem of signal extraction from pump noise,

$$
\begin{aligned}
& S(\omega)=\frac{P_{A}(\omega)-H(\omega) P_{B}(\omega)}{1-H(\omega) H(\omega)}, \\
& s(t)=f^{-1} S(\omega)=f^{-1}\left[\frac{P_{\mathrm{A}}(\omega)-H(\omega) P_{\mathrm{B}}(\omega)}{1-H(\omega) H(\omega)}\right],
\end{aligned}
$$

where

- $s(t)$ and $S(\omega)$ is the signal received by sensor A at time $t$ and its frequency domain transformed.

- $P_{\mathrm{A}}(\omega)$ and $P_{\mathrm{B}}(\omega)$ Fourier transformed pressure responses at sensors A and B, respectively.

- $f^{-1}$ is the inverse Fourier transformation.

- $H(\omega)=f^{-1} h(t)=G(\omega) e^{-j \omega \tau}$ is the Fourier transformed impulse response, $h(t)$, data transmission between sensor A and sensor B.

These researches provide a novel mud pulse signal detection and extraction techniques submerged into mud pump noise, attenuation, reflections, and other noise signals as it moves through the drilling mud. 


\section{MPT technology advances}

Significant technological advances have been made since the commercialization of LWD in 1978. The increased abundant amount of downhole collected information has considerably encouraged oilfield service providers to research and develop faster real-time data rate equipment (Emmerich et al. 2015). The recent major changes in drilling technologies are the consequences of increasing demand for sophisticated LWD services. The demand has witnessed new developments of downhole and surface signal encoding and decoding techniques, data compression methods and improved signal processing techniques (Wassermann et al. 2009). Mud channel drilling characteristic variations have led LWD researchers to develop methods and techniques that overcome restrictions in improving the mud pulse system for safety and drilling optimization.

This section review development trends reflecting to MPT technologies and processes improvement with a focus on data rate transmission capacity, data compression, signal encoding and decoding techniques, software automation and hardware changes on conventional MPT pulsar system.

\section{MPT data rates and data compression}

In LWD logging, band-limited telemetry systems are required to collect and carry large amounts of downhole data and send them to surface components. The increased amount of information results into prolonged rig time that sacrifices the borehole information and limits the oilfield operation requirements (Jia et al. 2011; Zhang et al. 2008). Traditional mud pulse systems generated data rate ranging between 1 and 3 bps while the required transmission rate which could fit the current drilling demand was 4-6 bps (Shao et al. 2017). Initial novel research on continuous mud pulse telemetry waves attained the maximum data transmission of more than $10 \mathrm{bps}$ (Hutin et al. 2001). In 2008, new system was tested which managed to achieve data rates of up to $20 \mathrm{bps}$ and a minimum of 5 bps in weakly deteriorated channel conditions (Klotz et al. 2008b).

Effective and robust signal transmission techniques combined with data compression methods can improve the performance of MPT systems data rate transmission. The introduction of data compression techniques into mud pulse logging data (Wu 2008) can improve the MPT information quantity and quality to satisfy exploration and drilling information. Real-time logging data compression method and mud signal differential pulse-code modulation (DPCM) scheme can reduce data redundancy, achieve good signal decoding quality and low algorithm complexity (Yu et al. 2010; Zhang et al. 2009). With the compression ratio of at least $50 \%$, it can provide the alterable compressing ratio advantages, excellent decode quality and low algorithm complexity (Zhang et al. 2009). Half compressed real-time logging data can double the transmission efficiency of the LWD system when the error between the compressed and original data is less than 5\% (Yu et al. 2010). The 4 bps physical data transmission link with data compression addition can achieve effective data rates of up to $33 \mathrm{bps}$ at higher telemetry frequencies $(\geq 10 \mathrm{~Hz})$ (Caruzo et al. 2012). Implemented Orion II telemetry platform employed data compression algorithms that increased the quantity of data rate to $120 \mathrm{bps}$ (simulated results), providing real-time geo-steering drilling efficiency to surface from 12,300 m downhole (Gilles 2009). However, (Gilles 2009) and (Caruzo et al. 2012) did not disclose the data compression methods used. The data compression algorithms to maximize the effective data rate and allow delivery of real-time image data of equivalent quality were demonstrated by (Cooper et al. 2015). At surface, compatible data decompression algorithms are required to maintain compressed telemetry data quality.

\section{Signal modulation techniques}

The effective data rate can be increased by the use of modulation scheme with low bit error rate. To improve the mud pulse telemetry signal-to-noise ratio (SNR), the system that supports two signaling types, different signal modulations and various signal frequencies is required. Klotz et al. (2008c) introduced a self-adjusting processing algorithms which can switch carrier frequencies between baseband modulation (ASK, PSK and FSK) with underlying pulse position encoding whenever mud signal distortion and changes occur. Enabling efficient transmission, an innovative improvements to MPT in ultra-deep environment which use QPSK modulation scheme was tested by (Caruzo et al. 2012). To increase physical data rate, multiple bits were sent on a single pressure symbol to minimize transmission bit errors suffered in discrete modulation scheme. Mud pulse position coding techniques can improve signal coding efficiency and increase telemetry data rate speed. Cooper et al. (2015) encoded downhole negative pressure pulse signal using differential pulse position modulation (DPPM) scheme while (Shearer 2010) proposed a combination of pulse position modulation (PPM) and pulse width modulation (PWM) techniques to convey mud pulse position of both upward and downward transitions. In certain pulse variations, pulse amplitude modulation (PAM) can be used alone or in combination with PPM and/or PWM, allowing data to also be conveyed by the pulse amplitude.

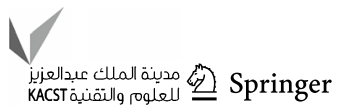


The use of these modulation schemes requires weaker signal detection method at the surface receiver, noise cancellation methods, compatible decompression algorithm and demodulation techniques to improve system quality.

\section{Hardware changes}

Several hardware changes in the conventional MPT systems have been proposed to enhance noise signal immunity to a minimum level. The use of an oscillating shear valve instead of rotational valve capable of generating pressure pulses at the appropriate frequency for the well conditions and the use of two surface pressure transducers on the surface have been tested (Klotz et al. 2008a, b, c). Surface noise cancellation algorithms in real-time to handle complex and continuously varying properties of the transmission mud channel to optimize signal transmission were implemented, as shown in Fig. 2. The developed MPT system was robust in the presence of surface piping, borehole drilling parameters and mud changing properties (Klotz et al. 2008b). The new mud pulse telemetry system using the annular-venting pulsar technology to create negative pressure pulses in the borehole of the drill string was demonstrated by (Cooper et al. 2015). The system objective was to improve downhole power management, actuation of the valve, and valve durability, thereby increasing the strength and data rate of the system.

\section{Software automation}

Recently, new advanced mud pulse telemetry system which generate digitally controlled pulse signals and automatic self-adjusted surface system to achieve high degree of freedom using the novel mud pulsar mechanism are designed. High speed mud pulse telemetry system (HSMPT) transmitting data at high rates using the known training sequence (TS) to adapt improved signal processing by comparing between the sequence and the received signal was introduced by (Emmerich et al. 2015). The HSMPT was tested in oil field with ultra-deep water drilling operation application using water-based mud (WBM) and high-viscosity oil-based mud (OBM) systems.

Later, research by (Emmerich et al. 2016a) improved the detection of the TS to automatically adjust adaptive filter to achieve the physical data rates of 10bits/s. The system was automatically able to tune the surface adaptive filters to any mud property changes that affect the channel signal characteristics, Fig. 3.

Challenges of the reliable automatic TS detection in severe harsh environments introduced additional changes to the overall system where new noise-reduction algorithm was introduced in the detection method, Fig. 4 (Emmerich et al. 2016b). The adaptive filter coefficients were automatically tested in real time and demonstrated good performance indicator application.
Fig. 2 Signal processing of new MPT system (Klotz et al. 2008c)

Fig. 3 Adaptive filter update sequence of actions (Emmerich et al. 2016a)

Fig. 4 Improved HSMPT system (Emmerich et al. 2016b)
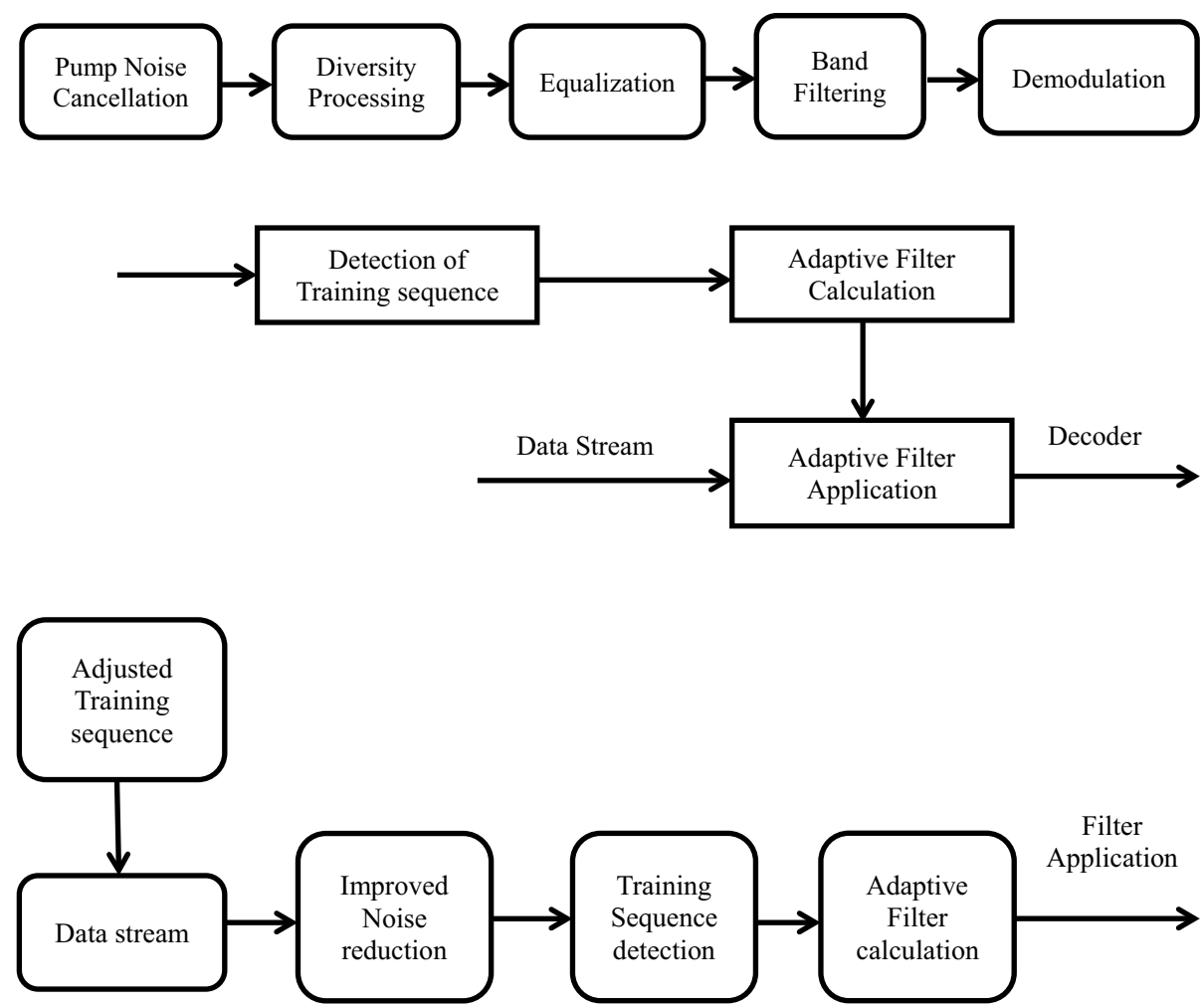
HSMPT operations experience specific environments drilling challenges caused by high-pressure or high-temperature and specific mud or certain rig construction limitations. The absence of reliable HSMPT created significant risks associated with health and safety that may cause accidents in the environment with inadequate critical downhole information (Emmerich et al. 2016a, b). Highly qualified and experienced personnel are required to fully operate HSMPT as the system components are very complex (Emmerich et al. 2016b). HSMPT systems are limited to field tests since their downhole signal conditions design are difficult and cannot be simulated in the laboratory environment (Emmerich et al. 2015). HSMPT creates additional materials and equipment's procurement logistics, repair and maintenance challenges as their designs are typically different to standard mud pulse telemetry. Convention improved mud pulse telemetry will still be the most used technology and economical solution for oil and gas exploration and drilling activities.

\section{Summary and discussion}

Petroleum exploration and drilling activities require high density logging data collections to ensure accurate drive and control operation. Precise formation evaluation and decision-making increases drilling field operation profits and economic value. Despite that wired coiled tubing telemetry technologies are technically efficient; they present numerous design and operational complexity. Mud pulse telemetry reliability and other incremental improvements contributed to be the best choice technology advantages. Mud pulse telemetry robust downhole signal modulation and data compression with surface noise cancellation algorithms have guaranteed immunity to noise signals and speed data rate transmission.

Researchers have shown key technical factors to improve mud pulse telemetry drilling performances are signal modulation technique, data compression methods, mud properties and surface noise cancellation techniques. Robust signal processing methods at the surface are imperative, but most of the schemes discussed amount to common sense procedures that can be difficult to implement. Detailed technical signal processes are not clearly disclosed, most of the journal papers mention general information to their solutions. Majority recommend subtracting, delaying and adding signals to create a type of weighted waveform that can improve signal-to-noise ratio (SNR) system performances. Oilfield service providers' patents and scientific papers are limited to field test operations and mostly kept as company secrets, making their methods difficult to simulate in the laboratory environment. LWD tools that are tested in one field situation can perform entirely differently in other fields. Measured standpipe signals can vary significantly from one rig to another without explanation; in some application extremely weak signals are found with tools that are mechanically functioning well.

Surface signal processing techniques recognize downhole coded and modulated signals generated by the poppet pulsar, siren pulsar or shear-valve pulsar under controlled pressure variations. Effective mud pulse telemetry signal modulation techniques combined with data compression methods can lower bit error rate, improve signal-to-noise ratio (SNR) and increase data rates transmission even under varying signal and mud properties distortions. Pulse position modulation (PPM) techniques and signal modulations which switch carrier frequencies between baseband modulations such as QPSK, DPSK and DBPSK generate robust signal-to-noise interferences. Properly designed filtering schemes and suitable derivation of mathematical models to reflect signal properties are significant. Adaptive finite impulse response (FIR) filters using least mean square (LMS) evaluation criterion are preferred to realize filter performances to eliminate or reduce various noise frequencies and recover mud pulse signals. Modified least mean square (LMS) algorithm has faster convergence speed, smaller steady state and lower excess mean square error which allow reasonable selection of filter step-size factor which relates to signal distortions.

Mud pulse telemetry system remains the most used technology in oil and gas exploration and drilling operations. Systems transmitting at more than 10 bps with higher data quality and transmission reliability can be simulated in the laboratory environment to match the real-time mounting demands.

\section{Conclusion}

Logging while drilling (LWD) environment poses extreme challenges in today's drilling activities of increasing operational risks and decreasing tolerance for errors. Surfacereceived signals are very noisy submerged into complex mud pump, interference, reflection, attenuation and random noise signal sources that weaken surface signal detection and extraction. Drilling decisions to obtain technology economic value have increased downhole information quantity and quality requirements which stimulated greater bandwidth and higher data rate transmission demands. Accurate downhole information signal impairment suppression methods prior to signal decoding are compulsory for safety reasons. Sophisticated digital signal and noise processing are compulsory to improve signal-to-noise ratio (SNR) performances. Logging tools must implement robust signal processing methods such as signal modulation, data compression method, surface noise cancellation techniques, surface signal demodulation and compatible data decompression algorithms to guarantee data quality and high data rates. 
Mud pulse telemetry signal processing techniques have improved drilling steering control, logging data measurements, formation evaluation accuracy and rigging time savings. Mud pulse technology step-change improvement demonstrates data transmission performance records, wide range operating environments and low operating cost. Surface signal processing techniques to cancel operational consequences of downhole mud pulses interacting with mud pump pressure signals which results into constructive or destructive interferences are compulsory.

Given the inherited mud pulse telemetry complexity with the working interaction surroundings, surface signal processing and noise cancellation methods are limited by certain assumptions and simplifications. Numerous researches provided contradicting details related to signal generation, signal transmission data rates, signal detection and extraction. It is apparent that a wide range of complex methods to enhance mud pulse telemetry signal operation effectiveness have been developed. Filtering schemes using adaptive FIR digital filter with least mean square (LMS) evaluation criterion have shown successful filter performances. More active noise cancellation design to evaluate least mean square (LMS), normalized least mean square (NLMS), recursive least square (RLS) and windowed adaptive FIR filter performances are open for future study. Filtering performance comparison between adaptive finite impulse response (FIR) and infinity impulse response (IIR) filters design may also be studied. More research on mud pulse transmission challenges, solutions and strategies are still open for further petroleum exploration and drilling efficiency.

Acknowledgements The authors wish to thank the anonymous reviewers for their valuable and constructive suggestions that improved this paper. This work was partly supported by the National Natural Science Foundation of China (Nos. 51604038, 51541408) and the Education Department of Hubei Province, China (D20141303).

Open Access This article is distributed under the terms of the Creative Commons Attribution 4.0 International License (http://creativeco mmons.org/licenses/by/4.0/), which permits unrestricted use, distribution, and reproduction in any medium, provided you give appropriate credit to the original author(s) and the source, provide a link to the Creative Commons license, and indicate if changes were made.

\section{References}

Akimov ON, Baule A, Bethge J, Roessel T, Tilsley-Baker R, et al (2006) Real-time transmission of high resolution images. In: SPE Europec/EAGE annual conference and exhibition. Society of petroleum engineers

Ali TH, Sas M, Hood JA, Lemke SR, Srinivasan A, McKay J, Mondragon C, Townsend SC, Edwards S, Fereday KS et al (2008) High speed telemetry drill pipe network optimizes drilling dynamics and wellbore placement. In: IADC/SPE drilling conference. Society of petroleum engineers
Blanco D, Rahimov K, Livescu S, Garner L, Vacik L et al (2016) Coiled tubing telemetry system improvements with real-time tension, compression, and torque data monitoring. In: Abu Dhabi international petroleum exhibition \& conference. Society of petroleum engineers

Blanco D, Sach M, Livescu S et al (2017) 3 1/2-in. Coiled tubing telemetry system saves time and improves operational efficiency during a multi-well campaign in Norway. In: SPE/ICoTA coiled tubing and well intervention conference and exhibition. Society of petroleum engineers

Brackel H-U (2016) Pump noise reduction and cancellation. US20140017092

Brandon TL, Mintchev MP, Tabler H et al (1999) Adaptive compensation of the mud pump noise in a measurement-while-drilling system. SPE J 4:128-133

Brooks AG (2015) Mud pulse telemetry noise reduction method. US $9,007,232 \mathrm{~B} 2$

Bultheel A (2003) Wavelets with applications in signal and image processing. Course Material University of Leuven, Belgium

Caruzo A, Hutin R, Reyes S, Tweel A, Temple P et al (2012) Advanced design and execution techniques for delivering high data rate MWD telemetry for ultradeep wells. In: OTC Arctic technology conference. Offshore Technology Conference

Chen W-Y, Fang B, Wang Y (2010) MWD drilling mud signal de-noising and signal extraction research based on the pulse-code information. In: Wavelet analysis and pattern recognition (ICWAPR), 2010 international conference on. IEEE, pp. 244-249

Cheng L, Jinfeng C, Zhao L, Shangchun F (2014a) Multiple-echo suppression modeling and experimental verification for acoustic transmission along periodic drillstring using dual receivers. Shock Vib 2014:295279

Cheng L, Jinfeng C, Zhao L, Shangchun F, Tianhuai D (2014b) Characteristics analysis of joint acoustic echo and noise suppression in periodic drillstring waveguide. Shock Vib. 2014

Cooper P, Santos LSB et al (2015) New mud pulse telemetry system delivers improved drilling dynamics and formation evaluation data. In: SPE Russian petroleum technology conference. Society of petroleum engineers

Cowan K (2005) Method for sealing lost circulation zones. US20050269085 A1

de Almeida Jr, IN, Antunes PD, Gonzalez FOC, Yamachita RA, Nascimento A, Goncalves JL (2015) A review of telemetry data transmission in unconventional petroleum environments focused on information density and reliability. J Softw Eng Appl 8:455

Emmerich W, Akimov O, Brahim IB, Greten A et al (2015) Reliable high-speed mud pulse telemetry. In: SPE/IADC drilling conference and exhibition. Society of petroleum engineers

Emmerich W, Akimov O, Brahim IB, Greten A et al (2016a) Field performance of automated high-speed mud pulse telemetry system. In: IADC/SPE drilling conference and exhibition. Society of petroleum engineers

Emmerich W, Greten A, Brahim IB, Akimov O et al (2016b) Evolution in reliability of high-speed mud pulse telemetry. In: Offshore technology conference. Offshore Technology Conference

Farraj AK, Miller SL, Qaraqe KA (2013) Propagation measurements for acoustic downhole telemetry systems. In: SPE annual technical conference and exhibition. Society of petroleum engineers

Fulda C, Akimov O, Baule A, Bethge J, Roessel T, Tilsley-Baker R (2006) Real-time transmission of high-resolution images. In: SPE Europec/EAGE annual conference and exhibition (Vienna, Austria)

Garner L, Vacik L, Livescu S, Blanco D et al (2016) Operational improvements with the expansion of an intelligent coiled tubing system to include real-time tension, compression, and torque data monitoring. In: SPE/ICoTA coiled tubing and well intervention conference and exhibition. Society of petroleum engineers 
Gilles V (2009) Delivering data to drive drilling decisions. Schlumberger, next generation exploration, development and production suit, pp 20-23

Guan L, Du Y et al (2004) Review of the application of wavelet analysis in the petroleum industry. In: Canadian international petroleum conference. Petroleum society of canada

Hahn D, Peters V, Rouatbi C, Scholz E (2008) Reciprocating pulser for mud pulse telemetry. US 7,417,920 B2

Harrell J, Brooks AG, Morsy HS (2000) Method and apparatus for mud pulse telemetry in underbalanced drilling systems. US6097310 A

Harrison WH, Mazza RL, Rubin LA, Yost AB et al (1990) Air-drilling, electromagnetic, MWD system development. In: SPE/IADC drilling conference. Society of petroleum engineers

Hartmann A, Akimov O, Morris S, Fulda C et al (2012) Improving real-time image-data quality with a telemetry model. SPE Drill Complet 27:383-392

Hernandez M, MacNeill DW, Reeves M, Kirkwood AD, Ruszka JP, Zaeper R, Lemke SR et al (2008) High-speed wired drillstring telemetry network delivers increased safety, efficiency, reliability, and productivity to the drilling industry. In: SPE indian oil and gas technical conference and exhibition. Society of petroleum engineers

Hutin R (2012) Zero sum pressure drop mud telemetry modulator. US 2012/0146807 A1

Hutin R, Tennent RW, Kashikar SV et al (2001) New mud pulse telemetry techniques for deepwater applications and improved real-time data capabilities. In: SPE/IADC drilling conference. Society of petroleum engineers

Jia AX, Qiao WX, Ju XD, Che XH, Lu R, Wang RJ (2011) Effect test on compression algorithms of acoustic logging downhole data. Well Logging Technol 35:288-291

Jianhui Z, Liyan W, Fan L, Yanlei L (2007) An effective approach for the noise removal of mud pulse telemetry system. In: Electronic measurement and instruments, 2007. ICEMI'07. 8th international conference On. IEEE, pp. 1-971

Klotz C, Reckmann H, Wassermann I, Macpherson JD, Ortiz JA, Brooks AG (2007) System and method for measurement while drilling telemetry. US 2007/0189119 A1

Klotz C, Bond PR, Wassermann I, Priegnitz S et al (2008a) A new mud pulse telemetry system for enhanced MWD/LWD applications. In: IADC/SPE drilling conference. Society of petroleum engineers

Klotz C, Kaniappan A, Thorsen AK, Nathan E, Jahangir M, Lie L et al (2008b) A new mud pulse telemetry system reduces risks when drilling complex extended reach wells. In: IADC/SPE Asia Pacific drilling technology conference and exhibition. Society of petroleum engineers

Klotz C, Wassermann I, Hahn D et al (2008c) Highly flexible mud pulse telemetry: a new system. In: SPE Indian oil and gas technical conference and exhibition. Society of petroleum engineers

Li J, Reckmann H (2009) System and method for pump noise cancellation in mud pulse telemetry. US 7,577,528 B2

Li C-W, Mu D-J, Li A-Z, Liao Q-M, Qu J-H (2007) Drilling mud signal processing based on wavelet. In: Wavelet analysis and pattern recognition, 2007. ICWAPR'07. international conference On. IEEE, pp. 1545-1549

Li H, Meng Y, Li G, Zhu L, Li Y, Chen Y (2015) Effects of suspended solid particles on the propagation and attenuation of mud pressure pulses inside drill string. J Nat Gas Sci Eng 22:340-347

Lin Y, Kong X, Qiu Y, Yuan Q (2013) Calculation analysis of pressure wave velocity in gas and drilling mud two-phase fluid in annulus during drilling operations. Math Probl Eng. 2013

Liu K (2016) Adaptive noise cancellation for electromagnetic-whiledrilling system. In: Information science and control engineering (ICISCE), 2016 3rd international conference on. IEEE, pp. 1253-1256
Liu X, Bo L, YUE Y (2007) Transmission behavior of mud-pressure pulse along well bore**. Project supported by the national high technology research and development program of China (863 Program, Grant No. 820-Q-04). J Hydrodyn Ser B 19:236-240

Livescu S, Blanco DA, Vacik L, Kubicki K et al (2015a) Novel 2 1/8in. Real-time downhole data monitoring system for coiled tubing operations. In: SPE annual technical conference and exhibition. Society of petroleum engineers

Livescu S, Blanco DA, Vacik L et al (2015b) 2 1/8-in. Intelligent coiled tubing system improves operational efficiency. In: International petroleum technology conference. International petroleum technology conference

Lu Y, Tang J, Ge Z, Xia B, Liu Y (2013) Hard rock drilling technique with abrasive water jet assistance. Int J Rock Mech Min Sci 60:47-56

Manning MJ, MacCallum D, Macpherson J, Taylor D, Zaeper R, King M, Hart E, Quinn T, Lofts J, Daykin C et al (2007). Processing wired pipe LWD-FE data in real time-experiences and lessons learned. In: SPWLA 48th annual logging symposium, pp. 1-9

Marsh JL, Fraser EC, Holt AL Jr et al (1988) Measurement-whiledrilling mud pulse detection process: an investigation of matched filter responses to simulated and real mud pressure pulses. In: Petroleum computer conference. Society of petroleum engineers

Mokhtar M, Khaidir M, Yaakub MY, Mustaffa SE, Keong AH, Kasim MH, Wijoseno DA et al (2014). Unlocking marginal field potential via fiber optic enabled coiled tubing integrated solutions. In: IADC/SPE Asia Pacific drilling technology conference. Society of petroleum engineers

Namuq MA, Reich M, Bernstein S (2013) Continuous wavelet transformation: a novel approach for better detection of mud pulses. J Pet Sci Eng 110:232-242

Neff JM, Camwell PL et al (2007) Field test results of an acoustic telemetry MWD system. In: SPE/IADC drilling conference. Society of petroleum engineers

Nygard V, Jahangir M, Gravem T, Nathan E, Evans JG, Reeves M, Wolter H, Hovda S et al (2008) A step change in total system approach through wired drillpipe technology. In: IADC/SPE drilling conference. Society of petroleum engineers

Ramondenc P, Baez F et al (2013) Using fiber-optic-enabled coiled tubing to quantify fluid placement and optimize stimulation effectiveness during matrix stimulation treatments in carbonate reservoirs. In: SPE European formation damage conference and exhibition. Society of petroleum engineers

Reckmann H (2008) Downhole noise cancellation in mud pulse telemetry. US 2008/0074948 A1

Reckmann H (2014) Downhole noise cancellation in mud pulse telemetry. US $8,811,118$ B2

Reeves M, MacPherson JD, Zaeper R, Bert DR, Shursen J, Armagost WK, Pixton DS, Hernandez M, et al (2006) High speed drill string telemetry network enables new real time drilling and measurement technologies. In: IADC/SPE drilling conference. Society of petroleum engineers

Schnitger J, Macpherson JD et al (2009) Signal attenuation for electromagnetic telemetry systems. In: SPE/IADC drilling conference and exhibition. Society of petroleum engineers

Shao J, Yan Z, Han S, Li H, Gao T, Hu X, Wei C (2017) Differential signal extraction for continuous wave mud pulse telemetry. J Pet Sci Eng 148:127-130

Shearer ES (2010) Pulse signaling for downhole telemetry. US 2010/0188253 p A1

Shen Y, Zhang L, Zhang H, Su Y, Sheng L, Li L (2013a) Eliminating noise of mud pressure phase shift keying signals with a selfadaptive filter. Indones J Electr Eng Comput Sci 11:3028-3035

Shen Y, Zhang L-T, Cui S-L, Sheng L-M, Li L, Su Y-N, (2013b) Delay pressure detection method to eliminate pump pressure interference on the downhole mud pressure signals. Math Probl Eng. 2013

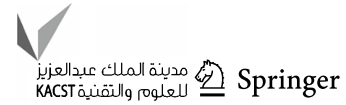


Shi Z, Liu X (2002) Multiphase technique improves mud pulse velocity calculations. Oil Gas J 100:45-45

Shi P, Brady D, Tennent R, Reyes SD (2004) Digital signal receiver for measurement while drilling system having noise cancellation. US6741185

Su Y, Sheng L, Li L, Bian H, Shi R, Chin WC (2011) High-data-rate measurement-while-drilling system for very deep wells. Houst. Tex. AADE

Taggart M, Murray N, Sturgeon T, et al (2011) New real-time data communication system enhances coiled tubing operations. In: Offshore mediterranean conference and exhibition. Offshore Mediterranean Conference

Wasserman I, Hahn D, Nguyen DH, Reckmann H, Macpherson J, et al (2008) Mud pulse telemetry sees step-change improvement with oscillating shear valves. Oil Gas J 106:39-39

Wassermann I, Reckmann H, Neubert M (2008) Estimation of properties of mud. US 2008/0002524 A1

Wassermann I, Kaniappan A, et al (2009) How high-speed telemetry affects the drilling process. J Pet Technol 61:26-29

Wolfe CA, Morris SA, Baule A, et al (2009) Enhanced real-time wellbore stability monitoring using high definition imaging with wired-pipe telemetry. In: SPE/IADC drilling conference and exhibition. Society of petroleum engineers

Wu JA (2008) Data compression technology and application. Science Press
Yu Z, Zhengding Q, Ke X, Shenghui W (2010) An algorithm for MWD data compression based on differential pulse code modulation. Pet Explor Dev 37:748-755

Yuan F, Gong X (2011) Research on signal processing of continuous wave mud pulse. In: Mechanic automation and control engineering (MACE), 2011 Second international conference On. IEEE, pp. 627-631

Zhang W, Shi YB, Wang ZG (2008) Wavelet neural network method for acoustic logging-while-drilling waveform data compression. J Univ Electron Sci Technol China 37:900-903

Zhang Y, Xiong K, Qiu ZD, Wang S, Sun D (2009) A new method for real-time LWD data compression. In: 2009 international symposium on information processing, pp. 163-166

Zhao Q, Zhang B, Hu H (2009) Novel two-step filtering scheme for a logging-while-drilling system. Comput Phys Commun 180:1566-1571

Publisher's Note Springer Nature remains neutral with regard to jurisdictional claims in published maps and institutional affiliations. 